\title{
Gravity, Entanglement and Spin Possible Timeless state of the Universe
}

\author{
Ahmed Farag Ali \\ Dept. of Physics, Faculty of Sciences, Benha University, Benha 13518, Egypt. \\ ahmed.ali@fsc.bu.edu.eg
}

\begin{abstract}
I localize gravity to match its measurements with the local inertial frame of special relativity. I find a geometric interpretation of the speed of light and mass. I find also a relation between every mass measured and the black hole entropy which introduces information-matter equation from gravity. Through localization of gravity, a timeless state of the universe emerges and the uncertainty principle does not hold since the velocity concept is replaced by distance in this timeless state. This would resolve the problem of time because timeless state of the universe emerges naturally and mathematically consistent. This would suggest that gravity form the hidden one variable of quantum mechanics which would complete the relation between quantum mechanics and gravity. The experimental evidence of timeless state of the universe is the quantum entanglement. Since the spin measurement is the manifestation of quantum entanglement. Therefore, the spin of quantum particle can be originated from geometrical or gravitational red-shift. We introduce also a principle of least computation which is achieved when the ratio equal to the difference in the process of local gravitational measurements.
\end{abstract}

\section{Localization of gravity}

Consider an existence of Schwarzschild black hole with event horizon. We consider the gravitational red-shift which is a property of general covariance.To localize gravity, we consider two points in the gravitational field of black hole as shown in Fig (1). Notice here this triangle follow the geodesics geometry of the considered black hole to connect the three points with each other. If $R$ and $A$ are far enough from $K$, the triangle become approximately Euclidean triangle.

Between these two points $A$ and $R$, there are two local measurements:

1. Relative gravitational red-shift which is represented by the ratio at two different slices

$$
\frac{z_{A}}{z_{R}}=\frac{\left(1-\frac{r_{s}}{r_{A}}\right)^{-1 / 2}-1}{\left(1-\frac{r_{s}}{r_{R}}\right)^{-1 / 2}-1}
$$

2. The difference in gravitational red-shift at two different slices or two different points.

$$
\Delta z=z_{A}-z_{R}=\left(1-\frac{r_{s}}{r_{A}}\right)^{-1 / 2}-\left(1-\frac{r_{s}}{r_{R}}\right)^{-1 / 2}
$$




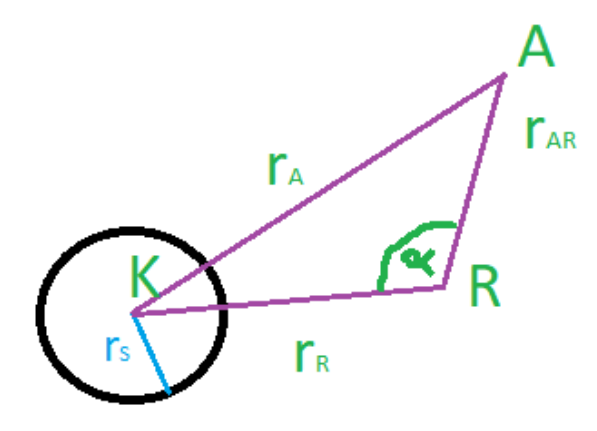

Figure 1: Black hole

\section{Matching local gravity measurement with local In- ertial frames}

\subsection{Relative gravitational red-shift}

In this section we match the local gravitational measurements with the local inertial frames. Therefore, we consider the weak gravitational approximations. We set $r_{s}<<r_{K}$ and $r_{s}<<r_{R}$. The gravitational red-shift for both $A$ and $R$ can be approximated as follows

$$
\begin{aligned}
& z_{A}=\left(1-\frac{r_{s}}{r_{A}}\right)^{-1 / 2}-1 \approx \frac{r_{s}}{2 r_{A}} \\
& z_{R}=\left(1-\frac{r_{s}}{r_{R}}\right)^{-1 / 2}-1 \approx \frac{r_{s}}{2 r_{R}} .
\end{aligned}
$$

We compute the relative gravitational red-shift using $\mathrm{Eq}(1.1)$. We express it in terms of all lengths measured at $R$ including the distance between $A$ and $R\left(r_{A R}\right)$.

$$
\frac{z_{R}}{z_{A}}=\frac{1}{\sqrt{1-\frac{r_{A R}^{2}}{r_{A}^{2}}+2 \frac{r_{R} r_{A R}}{r_{A}^{2}} \cos \alpha}}=\delta
$$

Notice the value of $\alpha$ can be $0 \leq \alpha \leq \pi / 2$. This equation represents the relative gravitational-red-shift between two points $A$ and $R$ in a weak gravitational field. For the case $\alpha=\pi / 2$. The relative gravitational red-shift will be given by

$$
\frac{z_{R}}{z_{A}}=\frac{1}{\sqrt{1-\left(\frac{r_{A R}}{r_{A}}\right)^{2}}}=\delta
$$

We notice the relative gravitational red-shift or relative gravitational time dilation matches with the definition of time dilation in special relativity $\left(1 / \sqrt{1-v^{2} / c^{2}}\right)$ if the ratio $r_{A R}^{2} / r_{A}^{2}$ can be replaced by ratio $v^{2} / c^{2}$ where $\mathrm{v}$ is the relative velocity and $\mathrm{c}$ is the speed of light. The match is legitimate and mathematically consistent since the relative gravitational red-shift introduces a local measurement which is the case for special relativity 
that holds only in local inertial frames. This means when $\alpha=\pi / 2$, the gamma factor of special relativity emerges as a ratio between the gravitational red-shift at $A$ and red-shift at $R$. Local gravity measurements depends only on "one variable"; the distance from the gravitational source, which is the reason for velocity ratios turned to be lengths ratios in this delta factor in Eq. (2.4). The ratio $r_{A R}^{2} / r_{A}^{2}$ can be considered as a geometric interpretation of the ratio $v^{2} / c^{2}$. This comparison can be written as

$$
\frac{r_{A R}}{r_{A}}=\frac{r_{A R} / t}{r_{A} / t}=\frac{v}{c}
$$

This would support the approach of time varying speed of light as a solution of cosmological puzzles that was suggested in [4].

Notice that time can be inserted easily in the previous equation as a "redundant variable" which can be interpreted that the state of matching local gravity measurements with local inertial frames would correspond to a possible timeless state of the universe which is consistent mathematically through the matching process that we performed.

To realize the effect of other values of angle $\alpha$ in weak gravitational field, we consider an approximation which is $r_{A R}<<r_{A}, r_{A R}<<r_{R}$. In that case, the delta factor in Eq. (2.3) is approximated as following

$$
\delta \approx 1-\frac{r_{R} r_{A R}}{r_{A}^{2}} \cos \alpha
$$

It is found that this equation matches with the derivative of Kepler equation.

$$
\frac{d M}{d E}=1-e \cos E
$$

where $M$ is the mean anomaly, $E$ is the eccentric anomaly, and $e$ is the eccentricity. In our approximation, the eccentricity $e$ is approximately equal to $r_{R} r_{A R} / r_{A}^{2}$, and $E$ refers to the angle $\alpha$. This once again give a geometric interpretation of Kepler equation from the relative gravitational red-shift.

\subsection{Difference in Gravitational red-shift}

In this section, we compute local measurement as difference in gravitational red-shift. For weak gravitational approximation, we get

$$
\Delta z=z_{R}-z_{A}=\frac{r_{s}}{2 r_{R}}-\frac{r_{s}}{2 r_{A}}
$$

Let us make an approximation as following $r_{A}=r_{R}+x$, where $x<<r_{A}$ and $x<<r_{R}$. In that case, Eq. (2.8) will be rewritten as follows. We use the value of Schwarzschild radius $r_{s}=2 G M / c^{2}$

$$
\Delta z M c^{2}=G M^{2} \frac{x}{r_{R}^{2}}
$$

where $G$, is the gravitational constant, $M$, is the black hole mass and $c$ is the speed of light. From Eq.(2.5), $c$ can be set to equal to $r_{A}$ if we take $t$ to be unity since we agree that $\mathrm{t}$ is a 
redundant factor through matching local gravity measurement with local inertial frames. We find that Eq. (2.9) can be arranged to take the following form

$$
\Delta z M=\Delta M=G M^{2} \frac{x}{r_{A}^{2} r_{R}^{2}}
$$

where $\Delta M=\Delta z M . \Delta M$ represents relative relation of mass between any two different points in the gravitational field. This would give a geometric representation for relativistic relation $m c^{2}$ in terms of the difference between different points to the black hole. We want to understand the physical meaning of the factor $G M^{2}$ in r.h.s of Eq. (2.10). When we look at Bekenstein-Hawking entropy equation

$$
S_{B H}=\frac{c^{3} A}{4 G \hbar}=\frac{4 \pi}{c \hbar} G M^{2}
$$

where $A=16 \pi\left(G M / c^{2}\right)^{2}$ stands for surface area of a black hole. We found that the factor $G M^{2}$ in r.h.s of Eq. (2.10) between any two different points can be expressed in terms of black hole entropy as follows

$$
\Delta z M=\Delta M=\frac{\hbar}{4 \pi} \frac{x}{r_{R}^{2} r_{A}} S_{B H}
$$

We assumed that time is a unity. let us consider this unit as the Planck time. This means that the Planck constant in previous equation can be replaced through the following process

$$
t_{p}=\sqrt{\frac{\hbar G}{c^{5}}}=1
$$

Since the Planck time is our unity, then c can set to be $r_{A}$. Therefore, the Planck constant in this geometric picture will be given by

$$
\hbar G=r_{A}^{5}
$$

This equation gives a geometric or gravitational interpretation of Planck constant when matching local gravity measurement with local inertial frames.

The relative mass between any two different points is therefore given by

$$
\Delta z M=\Delta M=\frac{1}{4 \pi G} \frac{x r_{A}^{4}}{r_{R}^{2}} S_{B H}=\frac{1}{16 \pi G} \frac{x r_{A}^{2}}{r_{R}^{2}} A
$$

We notice that the difference in gravitational red-shift gives an emergence of relative mass. The previous equation gives purely a geometric expression for the relative mass in terms of the gravitational source area of its full entropy.

It is experimentally proved that the difference in gravitational potential has an effect on the apparent weight of the $14.4-\mathrm{keV}$ ray of Iron (Fe) [2,3]. This may be an experimental support for the derived relation that connect the difference in gravitational red-shift and emergence of mass in this section. 


\section{Gravity and Uncertainty}

In previous sections, we have shown that the concept of velocity is replaced with the relative distance between any any two different points in the space time when we match local gravitational measurements with the local inertial frames. This would generate a timeless state of the universe that is mathematically consistent in connecting non-locality with locality. In that state, the gravitational measurements happens in terms of only one variable which is the distance from the gravitational source. Time variable at the matching process appear to be a redundant variable. Since time, and therefore velocity dissolve when the matching process happens, therefore there is no meaning to define uncertainty in this timeless state of the universe. We conclude that the distance from the gravitational source form the hidden variable of quantum mechanics, which would complete the connection between quantum mechanics and gravity in one unified theory, which is the timeless state of the universe. This may complete the picture that was introduced in EPR [1]

\section{Strong Gravity Case}

In strong gravity case, we can use Eqs. 1.1 and 2.8 without any approximation. These relations can be computed for any two points, and it gives a wide spectrum of measurements of relative gravitational red-shifts and masses in strong gravity field. In strong gravity field, the triangle will not be perfectly Euclidean but can be computed for every kind of measurement by knowing the length of this triangle.

\section{Least computations as the universal code of matter and gravity}

The computations of gravitational measurements will use less computations if the relative gravitational red-shift equal to the difference in gravitational red-shift. In that case, what can represent the ratio will certainly represent the difference, with one variable. To achieve the least computations, the local gravitational measurements should satisfy the following condition

$$
z_{A}-z_{R}=\frac{z_{A}}{z_{R}}
$$

Which can be solved in which $z_{A}=\phi^{2}$ and $z_{R}=\phi$ for any arbitrary variable $\phi$.

For weak gravitational approximation; this condition can be written as

$$
-\frac{r_{s}}{2}=\frac{r_{R}^{2}}{r_{A}-r_{R}}
$$

Or it can be expressed in terms of black hole mass as

$$
G M=-\frac{r_{A}^{2} r_{R}^{2}}{r_{A}-r_{R}}
$$


This equation seems to be a universal code of matter-gravity which is noted to be universal inverse proportionality between Matter and Gravity.

\section{Experimental quantum entanglement evidence of timeless state}

The only measurement that happens in the timeless state is the quantum entanglement between spin of elementary particles at two different points in the space. Therefore, it is mathematically consistent to consider spin degree of freedom of elementary particles as a a gravitational or geometrical component. We will relate the spin to the angle $\alpha$ for known quantum particles as follows:

- Higgs particle energy excitation corresponds to spin zero. This happens when the angle $\alpha=0$. In that case, the energy excitation will look like a straight line moving over a perfect sphere around the gravitational source.

- Gauge Bosons of spin 1. This would correspond to the state in which the angle $\alpha=\pi / 2$.

- Fermion correspond to spin $1 / 2$. The spin up can correpond to $\alpha>\pi / 2$ and spin down can correspond to $\alpha<\pi / 2$

- Fermions and Gauge bosons could be understood as a symmetry breaking of spin 0 state. Therefore, the value of $\alpha$ angle is the parameter that breaks the spherical symmetry.

- In that sense, gravity explain the origin of spin for elementary particles.

We interpret that as spin is a non-local completeness of local quantum theory which is related to gravitational source. The spin in that sense is the gravity effect or gravitational degree of freedom on every quantum particle.

\section{Conclusion}

We found that the complete and certain description of the universe comes with considering the matching state between local measurements in gravitational red-shift and local inertial frames. This means the certainty comes by considering the distance from gravitational source as the hidden one variable of any measurement process that we perform between two different points. Time as uncertainty and gravity as certainty introduce a complete description of our experience of the universe. We found that the timeless state explain the quantum entanglement and suggest that the spin of elementary particles are gravity coupling with the elementary particles. We introduced also the principle of least computations that introduce a relation between gravity and matter. 
Acknowledgment The author is deeply grateful for enlightening discussions with Klee Irwin, Raymond Aschiem and Hassan Alshal. This work is supported by the quantum gravity research grant, Los Angeles, California.

\section{References}

[1] A. Einstein, B. Podolsky and N. Rosen, Phys. Rev. 47, 777-780 (1935) doi:10.1103/PhysRev.47.777

[2] R. V. Pound and G. A. Rebka, Jr., Phys. Rev. Lett. 4, 337 (1960). doi:10.1103/PhysRevLett.4.337

[3] R. V. Pound and J. L. Snider, Phys. Rev. Lett. 13, 539 (1964). doi:10.1103/PhysRevLett.13.539

[4] A. Albrecht and J. Magueijo, Phys. Rev. D 59, 043516 (1999) doi:10.1103/PhysRevD.59.043516 [astro-ph/9811018]. 